\title{
Slow- and fast-light: fundamental limitations
}

\author{
ROBERT W. BOYD*† and PAUL NARUM† \\ $\uparrow$ The Institute of Optics, University of Rochester, \\ Rochester, NY 14627, USA \\ \$Norwegian Defence Research Establishment, Norway
}

(Received 19 March 2007; in final form 14 August 2007)

\begin{abstract}
We present an analysis of the propagation of light pulses through materials possessing extreme values of the group velocity. We begin with an analysis of the behaviour that occurs upon propagation through materials possessing simple Lorentzian gain or absorption lines or materials possessing sharp dips in gain or absorption features. We also describe how more complicated lineshapes can be used to tailor the dispersion of a slow-light system. We furthermore present an analysis of fundamental limitations to how many pulse widths a pulse of light can be delayed or advanced. We show how these mechanisms lead to different limits for slow and fast propagation media.
\end{abstract}

\section{Introduction}

In recent years, many researchers have been intrigued by the recently acquired ability to study light propagation under highly exotic conditions [1,2]. Equally exciting is the possibility that these techniques will lead to useful applications in the fields of telecommunications and electronics $[3,4]$. This paper is devoted to the examination of some fundamental aspects of slow- and fast-light propagation. In particular, we make a careful examination of the role of atomic resonances in achieving extreme values of the light propagation velocity, and we analyse some of the physical processes that can pose a limitation on how much a pulse of light can be delayed or advanced upon propagation through a resonant medium.

\section{Methods for controlling the speed of light}

We begin by making some general observations regarding several of the mechanisms that have been used to control the velocity of light.

We first note that the most dramatic modifications of the velocity of light are often those based on the exploitation of atomic resonances. The reason for this

\footnotetext{
*Corresponding author. Email: boyd@optics.rochester.edu
} 
behaviour is the following. The velocity with which pulses of light propagate through a material system is conventionally described by the group velocity

$$
v_{\mathrm{g}}=\frac{c}{n_{\mathrm{g}}},
$$

where the group index is given by

$$
n_{\mathrm{g}}=n+\omega \frac{\mathrm{d} n}{\mathrm{~d} \omega} .
$$

In these equations, $c$ is the speed of light in vacuum, $\omega$ is the angular frequency of the optical radiation, and $n$ is the usual refractive index. In essentially all slow-, fast- and backwards-light situations, the second term in the expression for $n_{\mathrm{g}}$ dominates. Thus, highly dispersive media are best suited for displaying extreme values of the group velocity, and sharp spectral features such as those encountered in atomic vapours are well suited for producing these effects. Note that $\mathrm{d} n / \mathrm{d} \omega$ can be either positive or negative. When the group index is large and positive, one speaks of slow light [5-12]; when the group index is either negative or positive and less than unity one speaks of fast light [13-18]. The special situation in which the group index is negative is sometimes known as backwards light. Backwards light is considered to be a form of fast light because, even though the peak of the pulse moves backwards within the material, the peak emerges from the material earlier than it would for propagation through an equal length of vacuum. Also intriguing is the observation that certain material systems can display either slow- or fast-light depending on the details of the experimental conditions [19, 20].

The simplest procedure for obtaining extreme values of the group velocity is to make use of the linear optical response of an isolated absorption or gain resonance. This situation is illustrated in figure 1. The case of an absorption resonance is

(1)

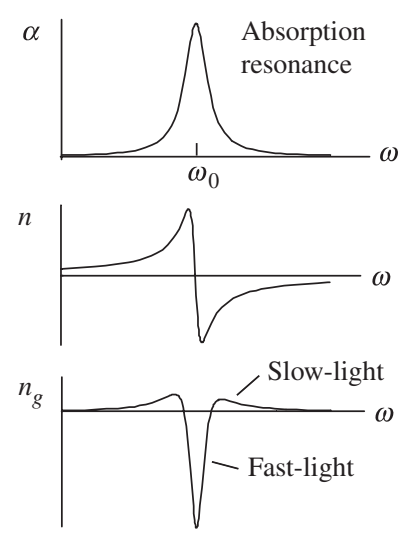

(b)

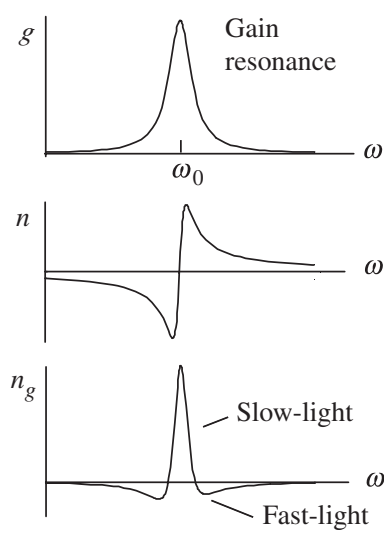

Figure 1. Slow- and fast-light based on isolated atomic resonances: (a) absorption resonance; (b) gain resonance. 
illustrated on the left of the figure and that of a gain resonance on the right of the figure. Associated with the absorption or gain resonance is a rapid frequency variation of the refractive index, as required by Kramers-Kronig relations, which is illustrated in the central panel on each side. Note that the sense of the frequency variation is exactly opposite in the two cases. The bottom panel on each side shows the resulting variation of the group index, as predicted by equation (2). Note that, for an isolated absorption resonance, fast-light occurs on line centre and slow-light occurs in the wings of the line; the situation is just the opposite for the case of a gain resonance. Because of the large attenuation that light experiences at the centre of a strong absorption resonance, it is difficult (but not impossible [13]) to observe fast light under these circumstances.

In most practical uses of slow- and fast-light it is necessary to be able to actively control the value of the group velocity. Nonlinear optical methods are often used to achieve this control of the group velocity. For example, electromagnetically induced transparency [9], coherent population oscillations [10], and saturated absorption [11] have been used to create narrow transparency windows in absorbing materials. The variation in refractive index associated with these absorption dips can be used to create slow- and fast-light. This situation is illustrated on the left-hand side of figure 2, with the analogous situation for a spectral hole in a gain medium illustrated on the right. As in figure 1, the central panels show the associated variation in refractive index and the lower panels show the variation of the group index. Again, in both cases either slow- or fast-light can be obtained depending on the detuning from line centre.

In certain applications of slow- and fast-light, it is desirable to tailor the dispersion properties to minimize the effect of pulse distortion. The procedure of Stenner et al. [12] is shown in figure 3. These researchers make use of a double gain line to flatten the gain profile of their material system. As a result, the refractive index profile has the form shown in the lower panel. Note that the region of nearly linear

(a)
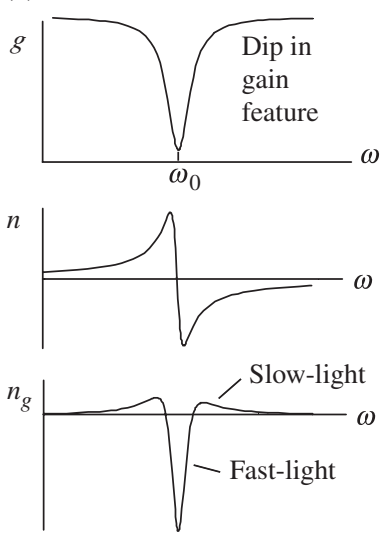

(b)
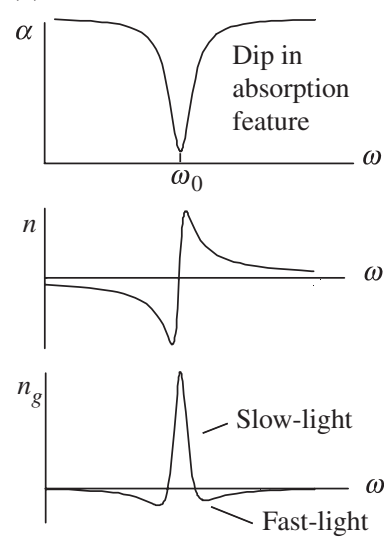

Figure 2. Slow- and fast-light based on a dip in $(a)$ a gain or $(b)$ absorption feature. 

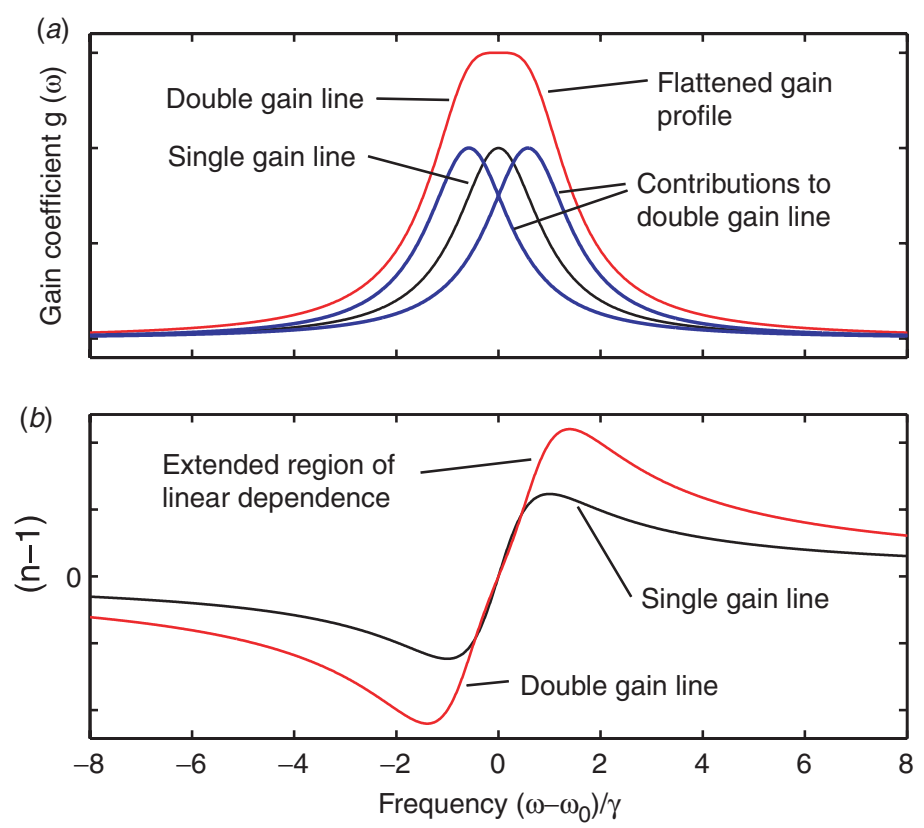

Figure 3. Example of disperson management in a slow-light system [12]. The use of a spectrally flattened, double gain line $(a)$ produces a broader spectral region of nearly linear dispersion $(b)$. (The colour version of this figure is included in the online version of the journal.)

dependence of index of refraction upon detuning is larger in this case than for a single gain line. As a result, short optical pulses, which necessarily possess a broad frequency spectrum, will suffer less distortion when propagating through the doublegain-line system.

\section{Fundamental limits on slow- and fast-light}

A key figure of merit for many slow- and fast-light systems is the number of pulse widths of delay or advancement that the system can produce. This number, which we will call the normalized delay or advancement, is equal to the number of pulses that can be contained at any time within the material and is a measure of the information storage capacity of the system. There is considerable conceptual and practical interest in determining what fundamental physical processes, if any, pose a limitation on how large this storage capacity can be [21-26]. In many situations, this storage capacity is limited by pulse distortion that will accompany large propagation distances. Pulse distortion is caused primarily by group velocity dispersion and spectral reshaping of the input pulse [23]. When working at or near line centre, the situation considered in the present paper, spectral reshaping, is the dominant mechanism. When operating far from line centre, group velocity dispersion is the dominant effect. 
As is well known, for a slow-light medium the pulse will tend to broaden in time upon propagation, whereas for a fast-light medium the pulse will tend to become narrower [22]. If the limit on pulse broadening is set to be a factor of $2^{1 / 2}$ and the limit on pulse narrowing is set so that to first order the pulse becomes infinitesimally short, we find that the maximum propagation distance is set by the following inequality:

$$
\alpha L \leq(\gamma T)^{2}
$$

Here $\alpha$ is the line-centre gain or absorption coefficient, $L$ is the length of the medium, $T$ is the pulse length, and $\gamma$ is the relevant linewidth, that is, the width of the isolated feature for the example given in figure 1 or of the dip in the overall profile for the example given in figure 2. The conclusion of equation (3) follows, for example, from the argument used to go from equation (13) to (14) in [23]. The maximum delay or advancement is obtained through use of this maximum value of $L$ and is given by

$$
\frac{\Delta T}{T}=\frac{1}{2\left(2^{1 / 2}\right)}(\alpha L)^{1 / 2} .
$$

For the case of a slow-light system, there appears to be no fundamental limits on the extent to which one can delay a pulse of light [23], although there are very serious practical problems $[24,25]$. Conversely, as we shall demonstrate below, for a fastlight system there seem to be limitations of a nearly fundamental nature that determine how much one can advance a pulse of light [22]. This limitation is often at most two pulse widths of advancement and in practice is often still more restrictive. The question then is raised as to why the two situations are so different.

At first glance, the answer to this question may appear to be almost obvious. Causality requirements, for example, limit how far the information content of a pulse of light can be advanced in time, but place no limit on how much it can be delayed. However, upon reflection one concludes that this explanation cannot be adequate, because causality places a restriction on the information velocity [15] but not on the group velocity, the quantity that we are concerned with here. Moreover, we have seen in the previous section that there appears to be an almost complete symmetry between slow- and fast-light interactions, at least at the level of the atomic response.

We believe that we have uncovered two key differences between slow- and fast-light situations that explain why the limitations on fast-light propagation are so much more restrictive than for slow-light media. These differences are described next.

(i) Restrictions based on maximum allowable gain or loss. First, we note that for the case of an absorbing medium, if the attenuation at the signal frequency is too large, the transmitted pulse will be too weak to be detectable. We arbitrarily choose a minimum intensity transmission of $T=\exp \left(-\alpha_{0} L\right)=$ $\exp (-32)$ at the signal frequency in our studies. The value 32 is chosen arbitrarily, but our conclusions do not depend critically upon the actual numerical value. For the case of a gain medium, the nature of the restriction is somewhat different. Here we require that the gain not be too large at any frequency, because if the gain is too large at some frequency the process of 
amplified spontaneous emission will occur at that frequency and deplete the gain of the material. We place a numerical restriction that the intensity gain can be no larger than $G=\exp (32)$, where again there is some arbitrariness in the choice of the value 32 . For the case of an isolated gain or absorption resonance, of the sort described in figure 1, and for line centre operation, these conditions place a limit on the amount of advancement or retardation that can be achieved, and this limit is never more than several pulse widths. This result follows from equation (4). For $\alpha L=32$, we see that the normalized delay (or advance) can be no larger than approximately 2 . This prediction is confirmed by the numerical results presented below.

Let us next consider the case of a dip in a gain or absorption profile, the situation illustrated in figure 2. For the case of a gain medium, we find the same restriction as those given in the previous paragraph, because there is a limit to the maximum amount of gain even in the distant wings of the dip. But the situation is different for a dip in an absorption line. Here the attenuation in the wings of the line can be arbitrarily large, as long as the loss at line centre is negligible. In this case, there is no limit on how large the propagation distance can be, and hence there is no limit on how large the total delay can become. But it should be noted that this 'trick' [23] works only for dips in absorption lines, not in gain lines. Thus, one can achieve arbitrarily large delay but not arbitrarily large advancement.

(ii) Restrictions based on spectral reshaping of the pulse. Spectral reshaping of the light pulse is the dominant limiting effect in most slow- and fast-light systems. This process also behaves differently for slow- and fast-light systems, as we

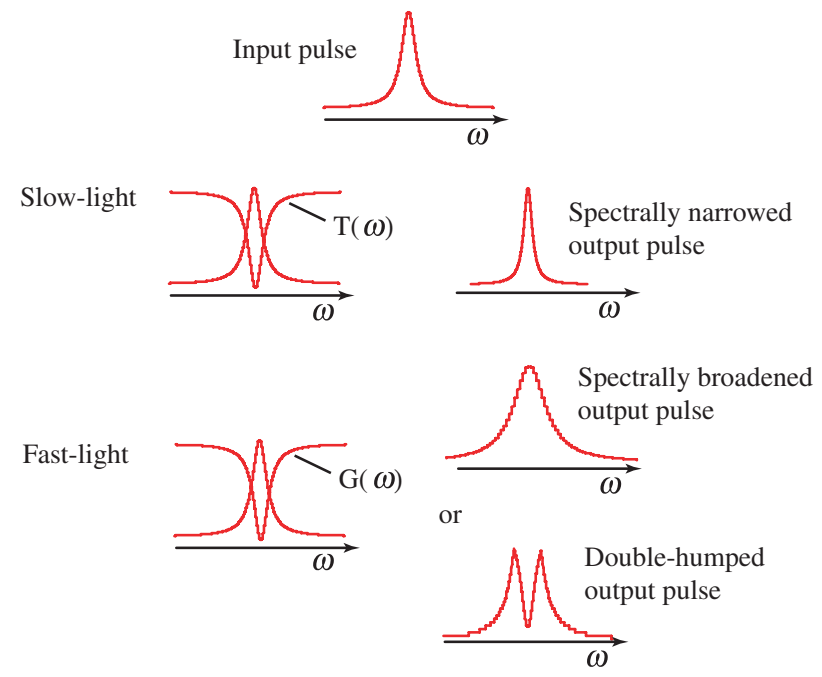

Figure 4. Influence of spectral reshaping of a pulse as it propagates through a slow- or fastlight system. Here $T(\omega)$ is the transmission spectrum and $G(\omega)$ is the gain spectrum of the material. (The colour version of this figure is included in the online version of the journal.) 
shall now see though use of figure 4. The spectrum of the input pulse is shown at the top of the figure. Under many practical situations, the spectral width of the pulse is comparable to the width of the transparency window. When this pulse propagates through a slow-light medium, the spectral wings of the pulse will experience lower transmission than the peak of the pulse. The pulse thus tends to become narrowed in frequency and hence broadened in time. This broadening is not desirable, but it is not as deleterious as what happens for the case of a fast-light medium, as illustrated near the bottom of the figure. In this case, the pulse broadens spectrally, and under certain conditions for long propagation distances the spectrum distorts so dramatically as to become multi-peaked. The pulse then shows ringing in the time domain. The onset of this severe form of pulse distortion determines the maximum possible extent of pulse advancement.

\section{Results of numerical modelling}

We have also performed a numerical study of the propagation of optical pulses through various slow- and fast-light media. Our procedure entails decomposing the input pulse (which is assumed to be a transform-limited Gaussian pulse) into a Fourier integral and allowing each frequency component to propagate according to

$$
E(\omega, z)=E(\omega, 0) \exp \{[\operatorname{in}(\omega) \omega / c-\alpha(\omega) / 2] z\}
$$

where $n(\omega)$ is the real part of the refractive index and $\alpha(\omega)$ is the absorption coefficient, both evaluated at frequency $\omega$. These quantities are calculated from the Lorentz oscillator model for each of the situations shown in figures 1 and 2 . These quantities thus obey causality (Kramers-Kronig) requirements. Finally, the time evolution of the output pulse is determined through an inverse Fourier transform. The results of this study are presented in figure 5. The fast light results are calculated for line-centre operation with a Lorentzian absorption line with an overall transmission of $\exp (-32)$. The slow-light results are calculated for line-centre operation with a Lorentzian gain line with an overall transmission of $\exp (+32)$. The amount of advancement and delay were controlled by varying the width of the resonance. For the slow-light case, we see that the pulse tends to broaden as the amount of delay is increased. For a delay of more than four pulse widths (of the initial pulse width), the amount of pulse broadening becomes excessive. For the fast-light case, we see that serious pulse breakup occurs for an advancement of two pulse widths.

\section{Summary and conclusions}

We have presented an analysis of the role of optical resonance in establishing slow and fast propagation of optical pulses. From the point of view of atomic response theory, there seems to be nearly complete symmetry between slow- and fast-light effects, and from this point of view it seems perhaps surprising that in practice it is 

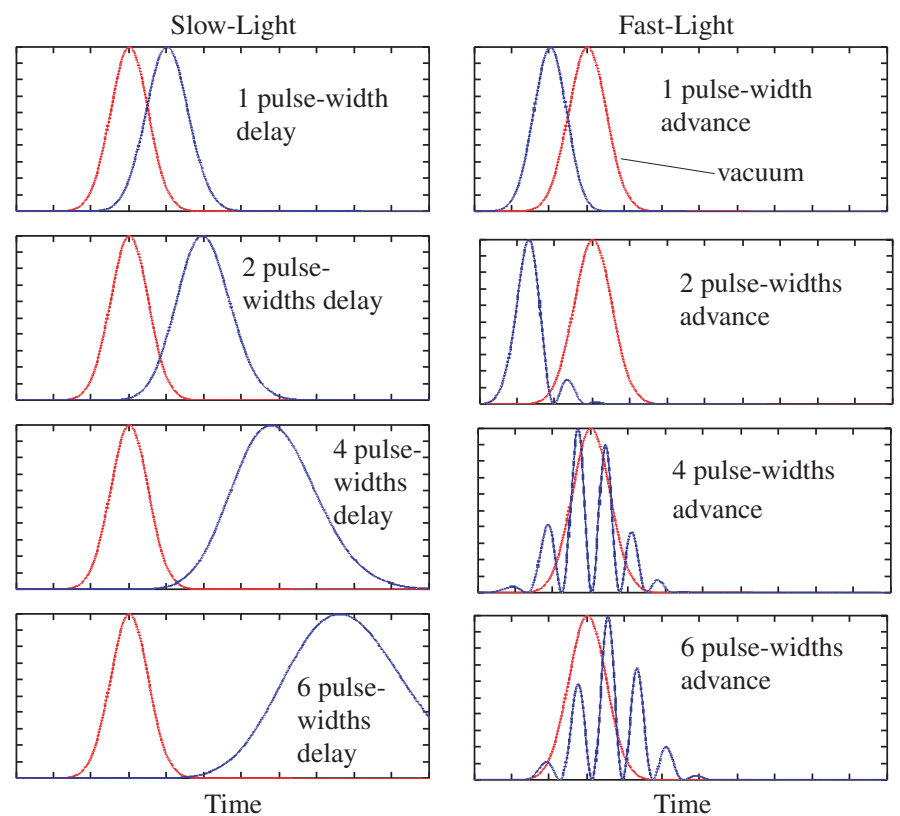

Figure 5. Numercial results illustrating the properties of pulse propagation through slowand fast-light media. Note that pulse broadening occurs for the slow-light case, whereas pulse breakup occurs for the fast-light case when one attempts to advance the pulse more than approximately two pulse widths. (The colour version of this figure is included in the online version of the journal.)

found to be easier to obtain large fractional delays than large fractional advances. We have identified two physical processes that lead to different behaviour of slowand fast-light media. One is that there is a maximum amount of attenuation that can be tolerated at the signal frequency for an absorbing medium, whereas there is a maximum amount of amplification that is allowable at any frequency for the case of a gain medium. The other process is spectral reshaping of the pulse, which also behaves differently for absorbing and amplifying media. We emphasize that the analysis of this paper is restricted to the situation in which the light is tuned to the centre of the resonance. Very large fractional delays have been observed in the far-detuned wings of absorption lines [11].

\section{Acknowledgements}

We gratefully acknowledge valuable discussions with George Gehring, Giovanni Piredda, Aaron Schweinsberg, Katie Schwertz, Zhimin Shi, Heedeuk Shin, Joseph Vornehm, and Petros Zerom. This work was supported by the DARPA/DSO Slow Light Program and by the NSF. 


\section{References}

[1] R.W. Boyd and D.J. Gauthier, in Progress in Optics, Vol. 43, edited by E. Wolf (Elsevier, Amsterdam, 2002).

[2] P.W. Milonni, Fast Light, Slow Light, and Left-Handed Light (Institute of Physics Publishing, Bristol, 2005).

[3] R.W. Boyd, D.J. Gauthier and A.L. Gaeta, Opt. Photon. News April 182006.

[4] D.J. Gauthier, A.L. Gaeta and R.W. Boyd, Photon. Spectra March 45 (2006).

[5] S.E. Harris, J.E. Field and A. Kasapi, Phys. Rev. A 46 R29, (1992).

[6] A. Kasapi, M. Jain, G.Y. Yin, et al., Phys. Rev. Lett. 742447 (1995).

[7] M.M. Kash, V.A. Sautenkov, A.S. Zibrov, et al., Phys. Rev. Lett. 825229 (1999).

[8] D. Budker, D.F. Kimball, S.M. Rochester, et al., Phys. Rev. Lett. 831767 (1999)

[9] L.V. Hau, S.E. Harris, Z. Dutton, et al., Nature 397594 (1999).

[10] M.S. Bigelow, N.N. Lepeshkin and R.W. Boyd, Phys. Rev. Lett. 90113903 (2003).

[11] R.M. Camacho, M.V. Pack and J.C. Howell, Phys. Rev. A 74033801 (2006).

[12] M.D. Stenner, M.A. Neifeld, Z. Zhu, et al., Opt. Express 139995 (2005).

[13] S. Chu and S. Wong, Phys. Rev. Lett. 48738 (1982).

[14] L.J. Wang, A. Kuzmich and A. Dogariu, Nature 406277 (2000).

[15] M.D. Stenner, D.J. Gauthier and M.A. Neifeld, Nature 425695 (2003).

[16] B. Segard and B. Macke, Phys. Lett. A 109213 (1985).

[17] B. Macke and B. Segard, Eur. Phys. J. D 23125 (2003).

[18] G.M. Gehring, A. Schweinsberg, C. Barsi, et al., Science 312985 (2006).

[19] M.S. Bigelow, N.N. Lepeshkin and R.W. Boyd, Science 301200 (2003).

[20] E.E. Mikhailov, V.A. Sautenkov, I. Novikova, et al., Phys. Rev. A 69063808 (2004).

[21] A. Kuzmich, A. Dogariu, L.J. Wang, et al., Phys. Rev. Lett. 863925 (2001).

[22] H. Cao, A. Dogariu and L.J. Wang, IEEE J. Select. Topics Quantum Electron. 952 (2003).

[23] R.W. Boyd, D.J. Gauthier, A.L. Gaeta, et al., Phys. Rev. A 71023801 (2005); ibid. 72 059903(E) (2005).

[24] A. Matsko, D. Strekalov and L. Maleki, Opt. Express 132210 (2005).

[25] J.B. Khurgin, Opt. Lett. 31948 (2006).

[26] M.S. Bigelow, N.N. Lepeshkin, H. Shin, et al., J. Phys. Condens. Matter 183117 (2006). 